Alexandre da Silva Galvão

\title{
INSTABILIDADE ESTÁTICA E DINÂMICA DE PÓRTICOS PLANOS COM LIGAÇÕES SEMI-RÍGIDAS
}

Tese de Doutorado

Tese apresentada ao Programa de Pós-Graduação em Engenharia Civil da PUC-Rio como requisito parcial para obtenção do título de Doutor em Engenharia Civil.

Orientadores: Paulo Batista Gonçalves

Ricardo Azoubel da Mota Silveira

Rio de Janeiro, 23 setembro de 2004 


\section{Alexandre da Silva Galvão Instabilidade Estática e Dinâmica de Pórticos Planos Com Ligações Semi-Rígidas}

Tese apresentada como requisito parcial para obtenção do título de Doutor pelo Programa de PósGraduação em Engenharia Civil da PUC-Rio. Aprovada pela comissão Examinadora abaixo assinada.

Prof. Paulo Batista Gonçalves

Orientador

Departamento de Engenharia Civil - PUC-Rio

Prof. Ricardo Azoubel da Mota Silveira

Co-orientador

Departamento de Engenharia Civil - Escola de Minas - UFOP

Prof. João Cyro André

Departamento de Engenharia de Estruturas e Fundações - USP -SP

Prof. Carlos Magluta

Programa de Engenharia Civil - COPPE - UFRJ

Prof. Marcelo Amorim Savi

Programa de Engenharia Mecânica - COPPE - UFRJ

Prof. João Luis Pascal Roehl

Departamento de Engenharia Civil - PUC - Rio

Prof. Raul Rosas e Silva

Departamento de Engenharia Civil - PUC - Rio

Prof. José Eugênio Leal

Coordenador Setorial do Centro

Técnico Científico - PUC - Rio

Rio de Janeiro, 23 setembro de 2004 
Todos os direitos reservados. É proibida a reprodução total ou parcial do trabalho sem autorização da universidade, do autor e do orientador.

\section{Alexandre da Silva Galvão}

Graduou-se em Engenharia Civil na UFOP (Universidade Federal de Ouro Preto) em 1996. Fez curso de Mestrado em Construções Metálicas no Programa de Pós-Graduação de Engenharia Civil da UFOP pelo qual recebeu título de mestre no ano de 2000.

Ficha Catalográfica

Galvão, Alexandre da Silva

Instabilidade estática e dinâmica de pórticos planos com ligações semi-rígidas / Alexandre da Silva Galvão; orientador: Paulo Batista Gonçalves; co-orientador: Ricardo Azoubel da Mota Silveira. - Rio de Janeiro: PUC, Departamento de Engenharia Civil, 2004.

v., 245 f.:il.;29,7 cm

1. Tese (doutorado) - Pontifícia Universidade Católica do Rio de Janeiro, Departamento de Engenharia Civil.

Inclui referências bibliográficas.

1. Engenharia Civil - Teses. 2. Instabilidade Estrutural. 3. Dinâmica Estrutural. 4. Não-linearidade geométrica. 5. Ligações semi-rígidas. 6. Pórticos Planos. I. Gonçalves, Paulo Batista. II. Silveira, Ricardo Azoubel. III. Pontifícia Universidade Católica do Rio de Janeiro. IV. Título. 
"A tua saudade corta mais que o fio de navaia.

$O$ coração fica aflito, bate uma e a outra faia.

E os olhos se enchem d'água que até a vista se atrapaia."

(do cientista e poeta Paulo Vanzolini)

Favelinha

Templo da juventude.

Ah a juventude!

energia sem remédio

que une sorrisos

de diferentes peitos

universos únicos e indivisíveis

diferentes culturas

existências frágeis e delicadas

diferentes paixões

confusões e medos velados

de muitos sotaques

máscaras que pesam nas faces dores disfarçadas

lembranças e saudades feridas de guerra

guerreiros da vida vivendo vivendo...vivendo...

fazendo, enfim, valer

num ruído irresponsável

delirante e universal

a humanidade.

(Favelinha é o nome que damos ao setor do prédio da PUC-Rio onde ficam nossas salas de estudos)

Dedico a todos que me fizeram, fazem e farão sofrer...

...de saudade... 


\section{Agradecimentos}

À PUC-Rio por me receber e me acolher durante esses 4 últimos anos.

Ao bendito apoio financeiro do $\mathrm{CNPq}$, sem o qual não realizaria este sonho.

A todos os professores que tive em minha vida, representados pela Irmã Fidelis do Educandário Santo Antônio de Ouro Preto que me alfabetizou, professor José Benedito Neves (in memoriam) da Escola Técnica Federal de Ouro Preto por ter mostrado tanto amor a esta linda profissão de educador, o meu pai João Galvão, meu professor por duas vezes na graduação, por ser a minha maior referência e meu maior incentivador, os meus professores do departamento de Engenharia Civil da UFOP, os meus professores da Puc-Rio.

À Ana Roxo, pela dedicação e amor ao seu trabalho e aos seus "filhos".

Aos meus orientadores por serem, sem nenhuma dúvida, duas das pessoas mais importantes em toda minha vida: o Prof. Paulo Batista Gonçalves pela amizade, paciência e enorme carinho com que me acolhe sempre, chegando mesmo a fazer papel de pai nos meus momentos mais difíceis; o Prof. Ricardo Azoubel da Mota Silveira, por sua dedicação por vezes inacreditável e pela forte amizade nestes 6 anos em que me acompanha.

Aos amigo Ivy Jeann pela grande amizade e constante companheirismo, mesmo à distância; Anderson e Janaína pelo enorme carinho com que sempre me recebem; Raissa, por me ensinar Francês e pela bonita amizade; Leonardo Pinheiro pela colaboração e exemplo de vida; Anderson Resende, pela energia contagiante e por ser um bom companheiro; Denilson Lucena, que por muitas vezes substituiu o papel do meu irmão aqui na PUC;

À linda família que fiz em Rio Piracicaba, pelo carinho e amor que me dedicam sempre. São grandes professores nesta matéria, o Amor.

Às pessoas que passaram a fazer parte da minha vida nestes últimos anos e que me fizeram sorrir, amar, AMAR, suspirar, chorar, que me provocaram medo, ansiedade, ilusões, desilusões, sonhos, enfim, encheram a minha vida de poesia e beleza... (e muitas reticências)...

À minha querida amiga Cris, que torce é reza por mim, me dedicando um carinho sem medidas.

Aos meus pais e ao meu irmão eu não agradeço, pois eles fazem parte de mim e, portanto, agradecem junto comigo cada um dos representados desta lista. 


\section{RESUMO}

Galvão, Alexandre da Silva; Gonçalves, Paulo Batista; Silveira, Ricardo Azoubel da Mota. Instabilidade estática e dinâmica de pórticos planos com ligações semi-rígidas. Rio de Janeiro, 2004. 245p. Tese de Doutorado - Departamento de Engenharia Civil. Pontifícia Universidade Católica do Rio de Janeiro.

O principal objetivo deste trabalho é o desenvolvimento de um programa computacional para a analise não-linear estática e dinâmica de pórticos planos com ligações flexíveis (semi-rígidas). Inicialmente é apresentada a metodologia de solução não-linear e as formulações dos elementos finitos adotados na base computacional implementada. Em seguida, são estudados vários exemplos de sistemas estruturais estáticos com caminhos de equilíbrio fortemente não-lineares com a finalidade de testar os programas implementados. Então é apresentada a formulação do problema dinâmico com a definição das equações diferenciais ordinárias de movimento e as expressões das matrizes de massa e amortecimento. A solução desse sistema de equações diferenciais ordinárias é obtida por métodos de integração numérica implícitos ou explícitos. Alguns destes métodos são apresentados neste trabalho e incorporados ao programa computacional em conjunto com estratégias adaptativas de incremento automático do intervalo de tempo de integração $\Delta \mathrm{t}$. Por fim, o sistema computacional desenvolvido é utilizado na modelagem e obtenção da resposta estrutural estática e dinâmica de alguns sistemas estruturais planos com comportamento eminentemente não-linear. Através destes resultados são analisados alguns fenômenos importantes de instabilidade estática e dinâmica, bem como possíveis mecanismos de colapso e a influência de parâmetros físicos e geométricos no comportamento estrutural.

\section{PALAVRAS-CHAVE}

Instabilidade estrutural; dinâmica estrutural; não-linearidade geométrica; ligações semi-rígidas; pórticos planos. 


\section{ABSTRACT}

Galvão, Alexandre da Silva; Gonçalves, Paulo Batista; Silveira, Ricardo Azoubel da Mota (advisors). Static and dynamic instability of plane frames with semi-rigid connections. Rio de Janeiro, 2004. 245p. D.Sc. Thesis Departamento de Engenharia Civil. Pontifícia Universidade Católica do Rio de Janeiro.

The main objective of this thesis is to develop a numerical methodology for the nonlinear static and dynamics analysis of plane frames with semi-rigid connections. Initially, the formulations of the adopted finite elements are presented and implemented together with numerical methodologies for the solution of the non-linear equilibrium equations. Then, some examples of strongly nonlinear structural systems under static loads are studied to check the methodology. Subsequently, the ordinary differential equations of motion are derived and the corresponding damping and mass matrices are presented. The solution of this system of ordinary differential equations is obtained by implicit or explicit numerical integration methods. Some of these methods are presented in this work and incorporated into the computational program together with adaptive strategies for the automatic increment of the time step $\Delta t$. Finally, the computational system here developed is used to study the static and dynamic response of some plain structural systems with an inherent nonlinear behavior. A detailed parametric study is carried out to identify the influence of physical and geometric parameters on the structural behavior. This enables the analysis of some important static and dynamic instability phenomena and identification of possible mechanisms of collapse.

\section{KEY-WORDS}

Structural instability; structural dynamic; geometric nonlinearity; semi-rigid connections; plane frames. 


\section{SUMÁRIO}

1 Introdução 11

1.1 Considerações iniciais e objetivos 11

$\begin{array}{ll}1.2 \text { Organização do Trabalho } & 13\end{array}$

1.3 Revisão Bibliográfica 14

2 Problema estrutural estático não-linear 20

2.1 Introdução 20

2.2 Formulação do elemento finito não-linear 21

2.2.1 Relações deformação-deslocamento 24

2.2.2 Incremento da energia potencial total 26

2.2.3 Elemento finito 28

2.2.4 Matriz de rigidez e vetor de forças internas 30

2.3 Formulação do elemento finito híbrido não-linear 33

2.4 Metodologia de solução não-linear 43 
13 Exemplos numéricos: análises estáticas não-lineares 48

3.1 Introdução 48

3.2 Arcos 50

3.2.1 Arco circular rotulado-engastado 50

3.2.2 Arco circular biarticulado $\quad 52$

3.2.3 Arco senoidal sob carregamento distribuído $\quad 55$

3.2.3.1 Estudo do parâmetro geométrico $\mathrm{z}_{0}$

3.3. Pórticos em $L \quad 64$

3.3.1 Pórtico de Lee 66

3.3.2 Pórtico de Roorda 67

3.3.3 Estudo paramétrico $\quad 70$

3.3.3.1 Influência das condições de apoio $\quad 71$

3.3.3.2 Influência da excentricidade do carregamento 73

3.3.3.3 Carregamento distribuído $\quad 75$

3.3.3.4 Influência da rigidez da ligação entre as barras 76

4 Problema estrutural dinâmico não-linear 79

4.1 Introdução $\quad 79$

4.2 Equações de movimento $\quad 79$

4.2.1 Considerações sobre a matriz de massa 82

4.2.2 Equações do movimento para o problema linear 85

4.2.3 Equações do movimento para o problema não-linear 86 geométrico

4.3 Análise de vibração livre $\quad 87$

4.3.1 Equação característica $\quad 87$

4.3.2 Algoritmo de solução do problema de autovalor 88

4.4 Algoritmos de integração 90

4.4.1 Métodos explícitos e implícitos 91

4.4.1.1 Método da diferença central 91

4.4.1.2 Método de Runge-Kutta 94

4.4.1.3 Método de Newmark 97

4.4.1.4 Método de Wilson- $\theta \quad 100$ 
4.4.2 Considerações sobre a análise dinâmica não-linear 104

$\begin{array}{ll}\text { 4.4.3 Adaptatividade no tempo } & 107\end{array}$

4.4.3.1 Estratégia adaptativa para métodos da família de Newmark 108

4.4.3.2 Estratégia adaptativa para o método Runge-Kutta 111

5 Exemplos numéricos: análises dinâmicas 114

5.1 Introdução 114

5.2 Exemplos envolvendo análise de vibração 114

5.2.1 Barras com ligações semi-rígidas 115

5.2.2 Arco senoidal sob carregamento distribuído 124

5.2.2.1 Arco abatido: $z_{0}=20 \mathrm{~mm} \quad 124$

$\begin{array}{ll}\text { 5.2.2.2 Estudo do parâmetro geométrico } z_{0} & 128\end{array}$

$\begin{array}{ll}\text { 5.2.3 Pórtico em L } & 136\end{array}$

5.3. Exemplos de validação dos algoritmos de integração 148

$\begin{array}{ll}\text { 5.3.1 Viga engastada-livre } & 148\end{array}$

5.3.2 Pórtico de Williams 151

5.3.3 Barras com ligações semi-rígidas 154

5.4 Exemplos numéricos de análise dinâmica não-linear 157

5.4.1 Arco senoidal 157

$\begin{array}{ll}\text { 5.4.2 Pórtico em L } & 168\end{array}$

$\begin{array}{ll}\text { 6. Conclusões e sugestões } & 173\end{array}$

$\begin{array}{lr}\text { 7. Referências } & 179\end{array}$

APÊNDICE A: Estratégias de incremento e iteração 197

APÊNDICE B: Modelos de ligação 206

APÊNDICE C: Programa Computacional 217 